\title{
Migration and Final Location of Hot Super Earths in the Presence of Gas Giants
}

\author{
Ji-Lin Zhou ${ }^{1}$ and Douglas N. C. Lin ${ }^{2,3}$ \\ ${ }^{1}$ Department of Astronomy, Nanjing University, Nanjing 210093, China \\ email: zhoujl@nju.edu.cn \\ ${ }^{2}$ UCO/Lick Observatory, University of California, Santa Cruz, CA 95064, USA \\ email: lin@ucolick.org \\ ${ }^{3}$ Kavli Institute of Astronomy and Astrophysics, Peking University, Beijing 100871, China
}

\begin{abstract}
Based on the conventional sequential-accretion paradigm, we have proposed that, during the migration of first-born gas giants outside the orbits of planetary embryos, super Earth planets will form inside the 2:1 resonance location by sweeping of mean motion resonances (Zhou et al. 2005). In this paper, we study the subsequent evolution of a super Earth $\left(m_{1}\right)$ under the effects of tidal dissipation and perturbation from a first-born gas giant $\left(m_{2}\right)$ in an outside orbit. Secular perturbation and mean motion resonances (especially $2: 1$ and $5: 2$ resonances) between $m_{1}$ and $m_{2}$ excite the eccentricity of $m_{1}$, which causes the migration of $m_{1}$ and results in a hot super Earth. The calculated final location of the hot super Earth is independent of the tidal energy dissipation factor $Q^{\prime}$. The study of migration history of a Hot Super Earth is useful to reveal it's $Q^{\prime}$ value and to predict its final location in the presence of one or more hot gas giants. When this investigation is applied to the GJ876 system, it correctly reproduces the observed location of GJ876d around $0.02 \mathrm{AU}$.
\end{abstract}

Keywords. Planets and Satellites: Formation; Celestial Mechanics

\section{Introduction}

The search for habitable planets is an essential step in the quests to unravel the origin of the Solar System and find life elsewhere. To date, more than 250 exoplanets are detected mainly by radial velocity survey of nearby solar-type stars $\dagger$. In the database, there are 17 planets with mass less than 25 Earth mass $\left(M_{\oplus}\right)$, and among them 8 planets have orbits with period $<10$ days. They are GJ876d, HD69830b, GJ674b, HD160691d, 55Cnc e, Gl581b, HD219828b and GJ436b. We call them 'hot super Earths'.

According to the conventional core-accretion scenario of planet formation, planets form in a protoplanetary disk around the host protostar. Through the sedimentation of dust, cohesive collisions of planetesimals, many embryos will form by accreting and clearing the planetesimals in their feed zone (a band centered on the embryo with a width of $\sim 10$ Hill radius) and result in dynamically isolated bodies. In a disk with several $\left(f_{d}\right)$ times of minimum mass solar nebular, the isolation mass is (Zhou et al. 2007),

$$
M_{\text {iso }}=0.51 \times 10^{-2} M_{\oplus} \eta k_{\text {iso }}^{3 / 2},
$$

where

$$
\begin{aligned}
& \eta=\left(f_{\mathrm{d}} f_{\text {ice }}\right)^{3 / 2}\left(\frac{a}{1 \mathrm{AU}}\right)^{3 / 4}\left(\frac{M_{*}}{M_{\odot}}\right)^{-3 / 2} . \\
& \log \left(k_{\text {iso }}\right)=\sqrt{b^{2}+0.61 c}-b \\
& b=2.8+0.33 \log \eta \\
& c=3.6+0.67 \log \eta+\log T_{\text {dep }},
\end{aligned}
$$

$\dagger$ http://vo.obspm.fr/exoplanetes/, http://exoplanets.org/. 
$f_{\text {ice }}=1$ for embryos inside the ice line and $f_{\text {ice }}=4.2$ outside that, $T_{\text {dep }}$ is the timescale of depletion of gas disk.

According to equation (1.1), the isolation mass inside the ice line $(a \approx 2.7 \mathrm{AU}$ in solar system) is to small to become a super Earth. Unless their nascent protostellar disks are highly compact, the observed super Earths are unlikely formed in situ. Some extra mechanisms are required to account for the excitation of eccentricity and the merge of isolated embryos into super Earths. In Zhou et al. (2005), we have proposed two mechanisms that may lead to the excitation of eccentricities of embryos: (1) During the type-II migration of a first-born gas giant planet outside the orbits of embryos, the locations of its mean motion resonances (mainly 2:1 resonance) sweep through the embryos region; (2) During the dispersal of the gas disk, the location of secular resonance between the gas giant and embryos sweeps through the inner orbits. Additional mechanisms have also been discussed by Raymond et al. (2007).

In this paper, we suppose a super Earth has formed through one of the above mechanisms, and study the subsequent evolution after the gas disk was depleted and the gas giant has stop its migration. First we briefly review the secular evolution of two planets under tidal dissipation. Then we show some numerical results in section 3 . Conclusions are presented in section 4 .

\section{Secular dynamics under tidal dissipation}

\subsection{Tidal perturbation timescale}

We adopt a two-planet system as a model. Suppose two planets with mass $m_{i}(i=1,2)$ (in the order from inner to outer) moving around a star with mass $m_{*}$ in the same orbital plane. Let $m_{1}$ be an Earth-like planet, and $m_{2}$ a gas giant, $S_{i}, \Omega_{i}, a_{i}, r_{i}$ are the radius, spin rate (with spin axis perpendicular to the orbital plane), semi major axis, distance from the star of planet $i(i=1,2)$, respectively. The acceleration to the relative motion of $m_{i}$ caused by the tidal interaction between the star and planet $m_{i}$ has the form of (Mignard 1979, Mardling \& Lin 2002)

$$
\mathbf{F}_{\mathrm{i}, \mathrm{tid}}=-\left(1+\lambda^{-1}\right) \frac{9 n_{i}}{2 Q_{i}^{\prime}}\left(\frac{m_{*}}{m_{i}}\right)\left(\frac{S_{i}}{a_{i}}\right)^{5}\left(\frac{a_{i}}{r_{i}}\right)^{8}\left[3 v_{i r} \hat{r}+\left(v_{i \phi}-r_{i} \Omega_{i}\right) \hat{\phi}\right],
$$

where $\hat{r}, \hat{\phi}$ are the unit vector of radial and transversal direction of the orbital plane, $\mathbf{V}_{\mathbf{i}}=v_{i r} \hat{r}+v_{i \phi} \hat{\phi}$ and $n_{i}$ are the Kepler velocity and mean motion of planet $i(\mathrm{i}=1,2)$, respectively, $Q_{*}^{\prime}$ and $Q_{i}^{\prime}$ are the effective tidal dissipation factor of the star and planet $i$ defined as $Q^{\prime}=3 Q /\left(2 k_{L}\right)$, where $Q^{-1}=\tan (2 \epsilon)$ is the effective dissipation function, $\epsilon$ is the tidal lag angle (Goldreich \& Soter 1966), $k_{L}$ is the Love number or twice the apsidal constance for gaseous planets, and

$$
\lambda=\left(\frac{Q_{*}^{\prime}}{Q_{i}^{\prime}}\right)\left(\frac{m_{*}}{m_{i}}\right)^{2}\left(\frac{S_{i}}{S_{*}}\right)^{5}
$$

is the ratio of tidal dissipation in the planet to that in the star. If $\lambda \gg 1$, tidal dissipation in the planet dominates the evolution.

The values $Q_{*}^{\prime}$ inferred form the observation of circularization period in various stellar clusters are $\sim 1.5 \times 10^{5}$ for young stars with age less than $0.1 \mathrm{Gyr}$, and $\sim 10^{6}$ for mature stars (Terquem et al. 1998, Dobbs-Dixon et al. 2004). The $Q^{\prime}$ value for Jupiter inferred form Io's orbit evolution ranges from $5 \times 10^{4}$ to $2 \times 10^{6}$ (Yoder \& Peale 1981). And for Earth, $Q_{E}^{\prime} \approx 60$ (Yoder 1995). Thus for a gas giant planet with Jupiter mass, suppose $Q_{*}^{\prime} \approx Q_{J}^{\prime}=10^{5}$, from Eq. (2.2), $\lambda \sim 10$, while for a terrestrial planet with Earth mass, $\lambda \sim 10^{4}$. So in the case of tidal interaction between an Earth-like planet and a star, tidal 
dissipation in the planet dominates the evolution, thus we neglect the contribution of tide in star in the following study.

Take $m_{1}$ as an example. Under the perturbation of tidal effect, the averaged equations (over a period of orbital motion) governing the evolution of planet $m_{1}$ are,

$$
\begin{aligned}
& <\dot{a}_{1}>_{\text {tide }}=-2 a_{1} \tau_{\text {tide }}^{-1}\left[f_{1}\left(e_{1}\right)-\left(\frac{\Omega_{1}}{n_{1}}\right) f_{2}\left(e_{1}\right)\right], \\
& <\dot{e}_{1}>_{\text {tide }}=-9 e_{1} \tau_{\text {tide }}^{-1}\left[f_{3}\left(e_{1}\right)-\frac{11}{18}\left(\frac{\Omega_{1}}{n_{1}}\right) f_{4}\left(e_{1}\right)\right], \\
& <\dot{\varpi}_{1}>_{\text {tide }}=<\dot{\lambda}_{1}>_{\text {tide }}=0 .
\end{aligned}
$$

where $\varpi_{1}, \lambda_{1}$ are the longitude of perihelion and mean longitude of the orbit of $m_{1}$ (with volume density $\rho_{1}$ ), respectively, and

$$
\begin{aligned}
\tau_{\text {tide }} & =\frac{4 Q_{1}^{\prime}}{63 n_{1}}\left(\frac{m_{1}}{m_{*}}\right)\left(\frac{a}{S_{1}}\right)^{5} \\
& =2.4 \times 10^{7} Q_{1}^{\prime}\left(\frac{a_{1}}{0.1 \mathrm{AU}}\right)^{\frac{13}{2}}\left(\frac{m_{*}}{m_{\odot}}\right)^{-\frac{3}{2}}\left(\frac{m_{1}}{m_{\oplus}}\right)^{-\frac{2}{3}}\left(\frac{\rho_{1}}{3 \mathrm{~g} \mathrm{~cm}^{-3}}\right)^{\frac{5}{3}} \mathrm{yr} .
\end{aligned}
$$

Functions used are:

$$
\begin{aligned}
& f_{1}(e)=\left(1+\frac{31}{2} e^{2}+\frac{255}{8} e^{4}+\frac{185}{16} e^{6}+\frac{25}{64} e^{8}\right) /\left(1-e^{2}\right)^{15 / 2}, \\
& f_{2}(e)=\left(1+\frac{15}{2} e^{2}+\frac{45}{8} e^{4}+\frac{5}{16} e^{6}\right) /\left(1-e^{2}\right)^{6}, \\
& f_{3}(e)=\left(1+\frac{15}{4} e^{2}+\frac{15}{8} e^{4}+\frac{5}{64} e^{6}\right) /\left(1-e^{2}\right)^{13 / 2}, \\
& f_{4}(e)=\left(1+\frac{3}{2} e^{2}+\frac{1}{8} e^{4}\right) /\left(1-e^{2}\right)^{5}, \\
& f_{5}(e)=\left(1+3 e^{2}+\frac{3}{8} e^{4}\right) /\left(1-e^{2}\right)^{9 / 2}, \\
& f_{6}(e)=\left(1+\frac{15}{7} e^{2}+\frac{67}{14} e^{4}+\frac{85}{32} e^{6}+\frac{255}{448} e^{8}+\frac{25}{1792} e^{10}\right) /\left(1+3 e^{2}+\frac{3}{8} e^{4}\right), \\
& f_{7}(e)=\left(1+\frac{45}{14} e^{2}+8 e^{4}+\frac{685}{224} e^{6}+\frac{255}{448} e^{8}+\frac{25}{1792} e^{10}\right) /\left(1+3 e^{2}+\frac{3}{8} e^{4}\right) .
\end{aligned}
$$

The evolution of spin rate $\Omega_{1}$ is subjected to,

$$
I_{1} \dot{\mathbf{\Omega}}_{1}=-\frac{m_{*} m_{1}}{m_{*}+m_{1}} \mathbf{r}_{1} \times \mathbf{F}_{1, \text { tide }}
$$

where $I_{1} \approx \frac{2}{5} m_{1} S_{1}^{2}$ is the inertial momentum of $m_{1}$. The averaged change rate is

$$
<\dot{\Omega}_{1}>_{\text {tide }}=\frac{5}{2} \tau_{\text {tide }}^{-1}\left(\frac{a_{1}}{S_{1}}\right)^{2}\left[f_{2}\left(e_{1}\right)-\left(\frac{\Omega_{1}}{n_{1}}\right) f_{5}\left(e_{1}\right)\right] .
$$

A stable equilibrium configuration occurs at

$$
\Omega_{1, e q}=\frac{f_{2}\left(e_{1}\right)}{f_{5}\left(e_{1}\right)} n_{1} .
$$

Since the timescale to reach the equilibrium state $\left(\sim \tau_{\text {tide }}\left(S_{1} / a_{1}\right)^{2}\right)$ is several orders less than the tidal circularization timescale, we suppose such a state is reached. Substitute Eq. (2.8) into (2.3), we derive the timescales of tidal evolution of $m_{1}$,

$$
\tau_{\text {a-tide }} \equiv \frac{a_{1}}{\dot{a}_{1}}=-\frac{\left(1-e_{1}^{2}\right)^{15 / 2}}{2 e_{1}^{2} f_{6}\left(e_{1}\right)} \tau_{\text {tide }}, \quad \tau_{\mathrm{e}-\text { tide }} \equiv \frac{e_{1}}{\dot{e}_{1}}=-\frac{\left(1-e_{1}^{2}\right)^{13 / 2}}{f_{7}\left(e_{1}\right)} \tau_{\text {tide }} .
$$

Note that, $\tau_{a-\text { tide }} \gg \tau_{e \text {-tide }}$ when $e_{1} \approx 0$. However, when $e_{1} \approx 1, \tau_{a-\text { tide }}$ and $\tau_{e-t i d e}$ could be very small, and $\tau_{a-t i d e}<\tau_{e-t i d e}$ as long as $e>0.63425 \ldots$...

Due to the huge difference of $Q^{\prime}$ between the Earth-like planet $m_{1}$ and the gas giant $m_{2}$, for our later investigation of tidal evolution with $a_{1}<0.63 a_{2}$, we neglect the tidal effect in planet $m_{2}$. 


\subsection{Secular evolution in the case of $e_{1} \ll e_{2}$}

When $e_{1} \ll e_{2}$, the secular evolution of $m_{1}, m_{2}$ under tidal dissipation and general relativity effect can be approximated by the following equations (Mardling 2006):

$$
\begin{aligned}
& \dot{e}_{1}=-W_{o} e_{2} \sin \eta-W_{T} e_{1} \\
& \dot{e}_{2}=W_{c} e_{1} \sin \eta \\
& \dot{\eta}=W_{q}-W_{o}\left(\frac{e_{2}}{e_{1}}\right) \cos \eta
\end{aligned}
$$

where $\eta=\varpi_{1}-\varpi_{2}, \alpha=a_{1} / a_{2}, \beta=\sqrt{1-e_{2}^{2}}$, and

$$
\begin{aligned}
& W_{o}=\frac{15}{16} n_{1}\left(\frac{m_{2}}{m_{*}}\right) \alpha^{4} \beta^{-5}, \\
& W_{T}=\tau_{e-t i d e}^{-1}, W_{c}=\frac{15}{16} n_{2}\left(\frac{m_{1}}{m_{*}}\right) \alpha^{3} \beta^{-4}, \\
& W_{q}=\frac{3}{4} n_{1}\left(\frac{m_{2}}{m_{*}}\right) \alpha^{3} \beta^{-3}\left[1-\sqrt{\alpha}\left(\frac{m_{1}}{m_{2}}\right) \beta^{-1}+\gamma \beta^{3}\right],
\end{aligned}
$$

with $\gamma=4\left(n_{1} a_{1} / c\right)^{2}\left(m_{*} / m_{2}\right) \alpha^{3}$, the ratio of general relativity to quadruple contribution of $\dot{\eta}$. According to these equations, the secular evolution of $e_{1}$ and $e_{2}$ mainly passes three stages:

(1) After a short time oscillation, the evolution of $e_{1}$ and $\eta$ reaches a state of librating around a quasi-equilibrium configuration with $e_{1}=e_{1}^{e q}$ and $\eta=2 n \pi$ or $(2 n+1) \pi$, where (Mardling 2006)

$$
e_{1}^{e q}=e_{2} \frac{W_{0}}{\left|W_{q}\right|}=\frac{5 / 4 \alpha e_{2}}{\beta^{2}\left|1-\sqrt{\alpha}\left(m_{1} / m_{2}\right) \beta^{-1}+\gamma \beta^{3}\right|} .
$$

(2) As $\eta$ librates and $e_{1}$ evolves to $e_{1}=0$ gradually, $a_{1}$ is damped according to Eq. (2.9), thus $m_{1}$ migrates inward efficiently.

(3) Finally $e_{2}$ is damped on a timescale $\tau_{c} \gg \tau_{e-t i d e}$. During this timescale, the orbit of $m_{1}$ is almost circularized, and migration of $m_{1}$ is effectively stop at a location $a_{1 f}$.

The location of $a_{1 f}$ is what we want to find. However, due to the presence of resonance motion, the evolution of the two-planet system in real situation is more complicate, as we will show below.

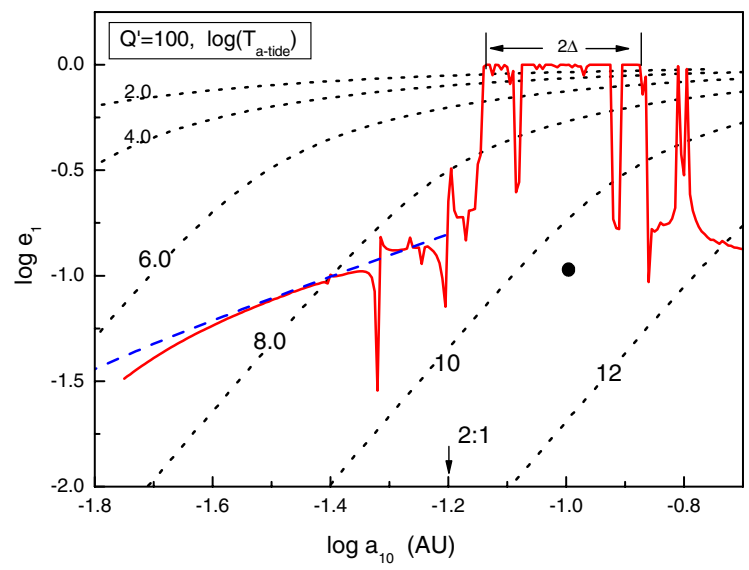

Figure 1. Maximum eccentricity (red solid line ) of $m_{1}$ in an initial circular orbit of semi-major axis $a_{10}$ excited by $m_{2}$ (black circle, with $a_{20}=0.1 \mathrm{AU}, e_{20}=0.1$ ). The black dotted lines with labels $2.0,4.0, \ldots$ denote the timescale $\left(\log \left(T_{a-t i d e} /\right.\right.$ years $\left.)\right)$ of $m_{1}$ from Eq. (2.9) at the specific location of $\left(a_{10}, e_{1}\right)$ with $Q_{1}^{\prime}=100$. The blue dashed line is obtained by two times of the equilibrium values defined by Eq. (2.12). 


\section{Numerical simulations}

We study the migration of an Earth-like planet under the tidal and mutual planetary perturbations with general three-body model. The system consists of a solar mass host star $\left(m_{*}=1 M_{\odot}\right)$, an Earth-like planet with mass $\left(m_{1}=5 M_{\oplus}\right)$, and a Jupiter mass gas giant $\left(m_{2}=M_{J}\right.$, where $M_{J}$ is Jupiter mass). Let $m_{1}$ be initially in a nearly circular orbit $\left(e_{10}=10^{-3}\right)$, and $m_{2}$ with initial elements $a_{20}=0.1 \mathrm{AU}, e_{20}=0.1$. To shorten the integration time, we let $Q^{\prime}=0.02$, as the migration timescale is proportional to $Q^{\prime}$.

By integration of the full equations of the general three-body system without tidal dissipation, we plot the maximum eccentricity $\left(e_{1 \max }\right)$ of $m_{1}$ excited by $m_{2}$ in Figure 1. The corresponding tidal-damping timescale obtained from equation $(2.9)$ with $\left(a_{10}, e_{1}\right)$ is also shown in the background of Fig. 1.

As we can see from Figure 1, the orbits of $m_{1}$ at most locations with $a_{10}<0.16$ AU have $T_{\text {a-tide }}<10 \mathrm{Gyr}$. However, most of those orbits in the Hill unstable region around $m_{2}$ with half width $\Delta=\left(e_{20}+2 \sqrt{3} h\right) a_{2} \approx 0.034 \mathrm{AU}\left(\right.$ where $h=\left[m_{2} /\left(3 m_{*}\right)\right]^{1 / 3}$ ) will be scattered to far away in our coplanar model. According to Zhou et al. 2005, embryos formed inside the location of the $2: 1 \operatorname{resonance}\left(a_{2: 1} \approx 0.063 \mathrm{AU}\right)$ with $m_{2}$ are dynamically stable, so we focus on the evolution of orbits with initial semi major axis $a_{10} \leqslant 0.063 \mathrm{AU}$.

If planet $m_{1}$ is initially located in lower order mean motion resonances with $m_{2}$, its eccentricity will be excited, thus a fast inward migration of $m_{1}$ is induced, according to Eq. (2.9). Fig. 2 shows the evolution of two orbits either from $2: 1$ resonance location $\left(a_{10}=0.063 \mathrm{AU}\right)$ or from non-resonance location $\left(a_{10}=0.040 \mathrm{AU}\right)$. During the subsequent passage through $5: 2$ resonance, the amplitude of eccentricity excitation is relative large. Recall that, according to Eq. (2.9), the $a$-damping timescale is much smaller than that of $e$-damping at high eccentricity. Thus a fast migration occurs until $e_{1}$ decrease to a small value $\sim 0.01$ (Novak et al. 2003). Then a slow migration linked with the secular dynamics occurs, with $\eta=\varpi_{1}-\varpi_{2}$ librating along an equilibrium value(see Fig. 2b).
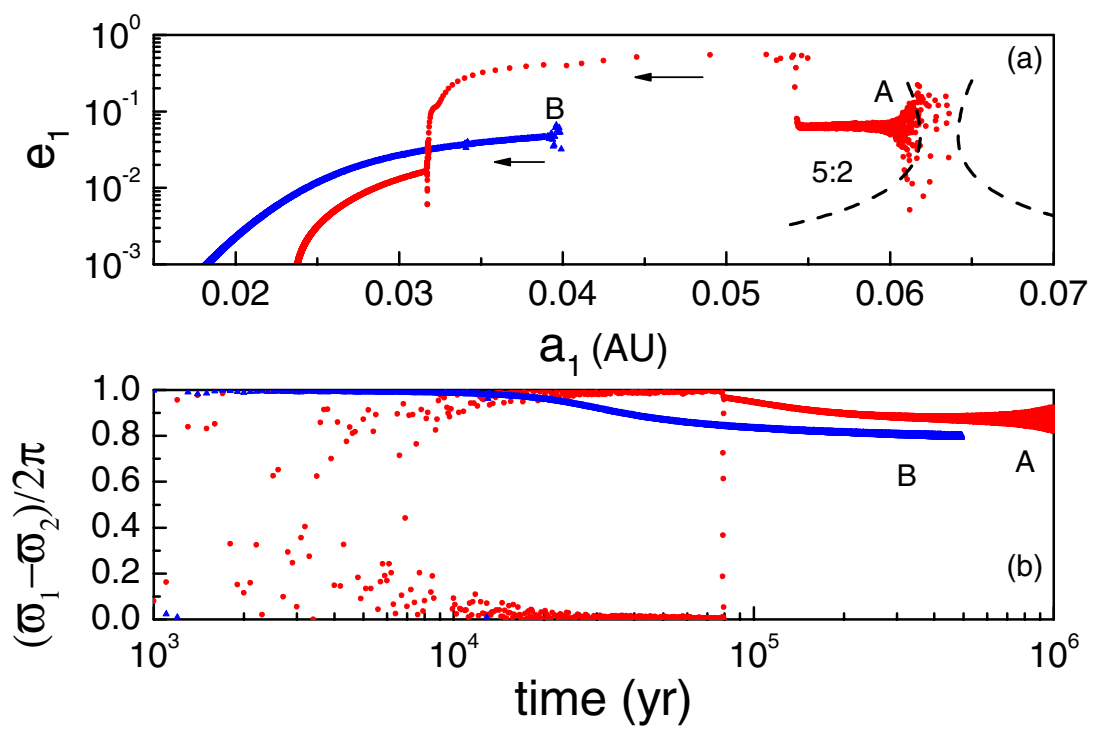

Figure 2. Evolution of orbits with $a_{10}=0.063$ (orbit A) and $a_{10}=0.040$ (orbit B). (a) Evolution track in $a_{1}-e_{1}$ plane. The dashed line shows the width of $2: 1$ resonance obtained from the circular restricted-three-body problem. The $5: 2$ indicates the $5: 2$ resonance location at the place that the eccentricity of orbits A jumps up. The arrows indicate the evolution directions. (b) Evolution of $\left(\varpi_{1}-\varpi_{2}\right)$ (in radian). 

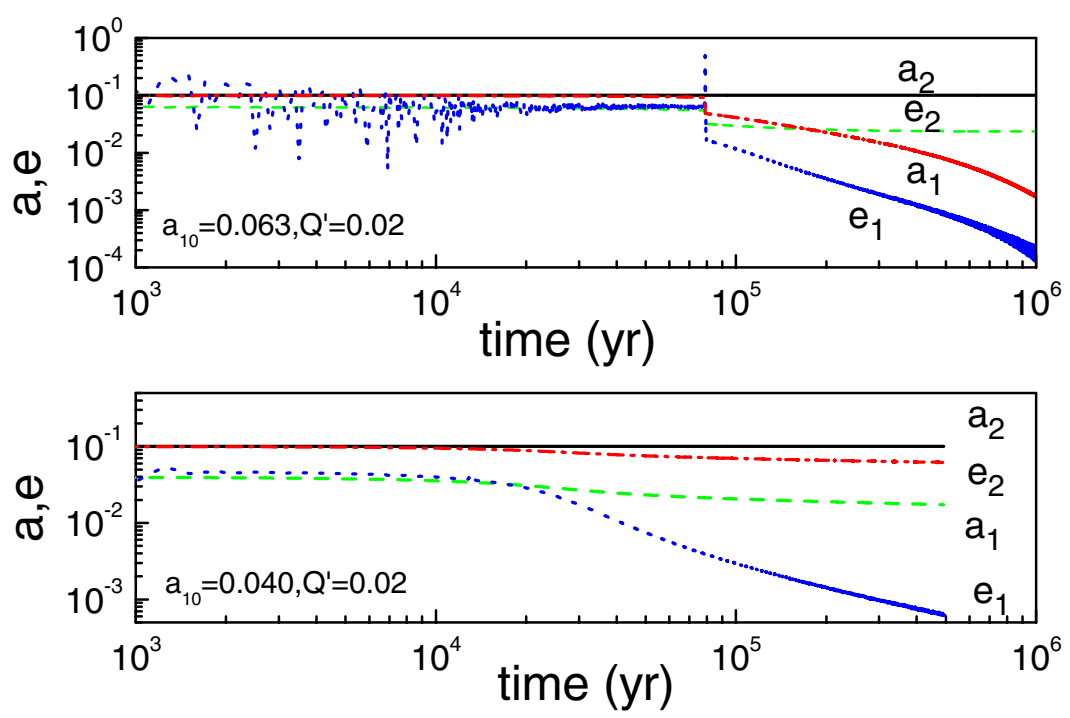

Figure 3. Evolution of orbital elements for the two orbits shown in Fig. 2 with initial elements $a_{10}=0.040,0.063$, respectively. The evolution of orbit with $a_{10}=0.040$ fits with the secular evolution described in section 2 , but for the orbit with $a_{10}=0.063$, the presence of the resonance leads to a dramatic increase (decrease) of $e_{1}\left(e_{2}\right)$ at time $t \approx 8 \times 10^{4}$ year, when the orbit crosses the $5: 2$ resonance in Fig. 2 .

The migration induced by resonant eccentricity-excitation is different from that excited by mutual secular perturbation. When we check the evolution of $a_{i}, e_{i},(i=1,2)$ during the passage of $5: 2$ resonance, we find that $a_{1}, e_{1}, e_{2}$ have dramatic decrease after the crossing the $5: 2$ resonance (Fig. 3). The decrease of $e_{2}$ causes the different final states (i.e., the final state of orbit $\mathrm{A}$ and $\mathrm{B})$ in $\left(a_{1}, e_{1}\right)$ plane of Fig. 2. The final location is around $0.018 \sim 0.025 \mathrm{AU}$, depending on the different evolutionary routines.

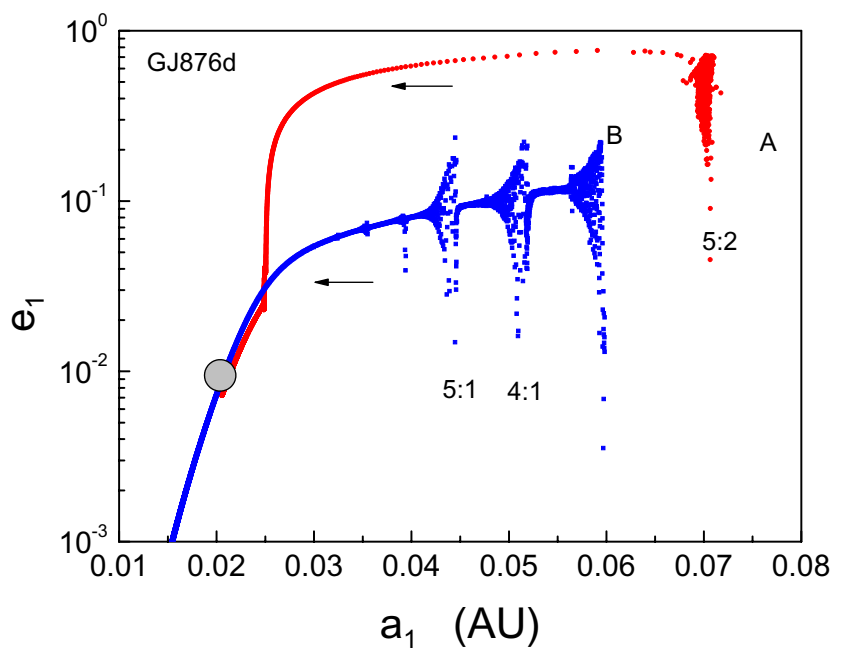

Figure 4. Evolution track of orbits with $a_{10}=0.07074$ (the exact location of $2: 1$ resonance, orbit $\mathrm{A}$ ) and $a_{10}=0.060$ (orbit $\mathrm{B}$ ). The arrows indicate the evolution directions. 
In order to show that the above track correctly reproduces the observed location of extrasolar planets, we applied this study to the GJ876 system. GJ876 is a M dwarf star located $4.72 \mathrm{pc}$ away from us in the solar neighborhood. To date, two gas giant planets, GJ876b and GJ876c, were observed to be located on orbits with period around 30 days and 60 days, an example of 2:1 mean motion resonance, and a hot planet GJ876d with mass around $5.7 M_{\oplus}$ in an orbit with period 1.94 days $(a=0.0208 \mathrm{AU}$, e=0). We numerical simulate the evolution of an Earth-like planet inside a gas giant located in the present orbit $\left(a_{20}=0.13 \mathrm{AU}, e_{20}=0.2243\right)$. Figure 4 shows the evolution track of $m_{1}$. The final location is around $0.02 \mathrm{AU}$ according to the simulation, which is almost independent of the initial location of $m_{1}$.

\section{Summary and discussions}

Many super Earths are observed to be located inside the orbits of gas giants. These super Earth and gas giant pairs may be a natural consequence of planet formation and migration. Embryos formed prior and interior to the gas giants are induced to migrate, collide, and evolve into close-in Super Earths (Zhou et al. 2005). In this report, we have shown that, the migration of super Earths under tidal dissipation and the perturbation from gas giants is mainly along the secular evolution paths. Although resonances between super Earths and gas giants may excite the eccentricity and speed the migration timescale, the final evolution path can be well determined.

According to the investigation of this paper, we find that the study of the evolution path provides useful information in the following ways: (i) the migration path shows the evolution history, especially the evolution of $e_{1}, e_{2}, a_{1}$ (Figs. 2, 4). (ii) Comparing the observed location of the planet in the path, we can deduce the range of $Q^{\prime}$ values for the hot Super Earths. We will investigate in more details on these topics in the future.

\section{Acknowledgements}

This work is supported by NSFC(10778603,10233020), National Basic Research Program of China(2007CB814800), NASA (NAGS5-11779, NNG04G-191G, NNG06-GH45G), JPL (1270927), NSF(AST-0507424, PHY99-0794).

\section{References}

Dobbs-Dixon, I., Lin, D. N. C., \& Mardling, R. 2004, ApJ, 610, 464

Goldreich, P. \& Soter, S. 1966, Icarus, 5,375

Ida, S. \& Lin, D. N. C. 2004, ApJ, 604, 388

Mardling, R. 2007, MNRAS(submitted), arXiv:0706.0224.

Mardling, R. \& Lin, D. N. C. 2004, ApJ, 573, 829

Mignard, F. 1979, The Moon and the Planets, 20, 301

Novak, G. S., Lai, D., \& Lin, D. N. C. 2003, in Scientific Frontiers in Research on Extrasolar Planets, eds D. Deming \& S. Seager, (San Francisco:ASP), 177

Raymond S., Barnes, R., \& Mandell A. M. 2007, arXiv:0711.2015.

Terquem, C. Papaloizou, J. C. B.,Nelson, R. P., \& Lin, D. N. C. et al. 1998, ApJ,502,788.

Yoder C. F. 1995 in Global Earth Physics, A Handbook of Physical Constants, ed. T. Ahrens (American Geophysical Union, Washington)

Zhou, J. L., Aarseth, S. J., Lin, D. N. C., \& Nagasawa, M. 2005, ApJ, 631, L85

Zhou, J. L., Lin, D. N. C., \& Sun Y. S. 2007, ApJ, 666, 423 